\title{
Method of lateral image reconstruction in structured illumination microscopy with super resolution
}

\author{
Qiang Yang, Liangcai Cao*, Hua Zhang, \\ Hao Zhang and Guofan Jin \\ State Key Laboratory of Precision Measurement Technology \\ and Instruments Tsinghua University, Beijing 100084, P. R. China \\ *clc@tsinghua.edu.cn
}

Received 30 August 2015

Accepted 26 October 2015

Published 23 December 2015

\begin{abstract}
The image reconstruction process in super-resolution structured illumination microscopy (SIM) is investigated. The structured pattern is generated by the interference of two Gaussian beams to encode undetectable spectra into detectable region of microscope. After parameters estimation of the structured pattern, the encoded spectra are computationally decoded and recombined in Fourier domain to equivalently increase the cut-off frequency of microscope, resulting in the extension of detectable spectra and a reconstructed image with about two-fold enhanced resolution. Three different methods to estimate the initial phase of structured pattern are compared, verifying the auto-correlation algorithm affords the fast, most precise and robust measurement. The artifacts sources and detailed reconstruction flowchart for both linear and nonlinear SIM are also presented.
\end{abstract}

Keywords: Microscopy; structured illumination; super-resolution; image reconstruction.

\section{Introduction}

Due to the finite aperture size in a lens imaging system, the information with high spatial frequencies will be lost by the diffraction of light wavefront, imposing an ultimate barrier in optical resolution to conventional optical microscopy. The achievable highest optical resolution, or the minimum resolvable distance between two objects in a conventional light microscope, is about $200 \mathrm{~nm} .^{1}$ To circumvent the diffraction limitation and achieve super-resolution image, in the past two decades a number of optical methods have been developed and got tremendous success by combining themselves with fluorescent probe techniques. Among those methods, stimulated emission depletion (STED) is based on depleting fluorescence emission in the peripheral area of a light focus, while stochastic optical reconstruction microscopy (STORM) and

${ }^{*}$ Corresponding author.

This is an Open Access article published by World Scientific Publishing Company. It is distributed under the terms of the Creative Commons Attribution 4.0 (CC-BY) License. Further distribution of this work is permitted, provided the original work is properly cited. 
photoactivated localization microscopy (PALM) rely on the localization of single fluorescence emitters. $^{2}$ Different from these methods, the essential concept of super-resolution structured illumination microscopy (SIM) to enhance image resolution is to encode undetectable high-frequency information into detectable low-frequency band by illuminating the specimen with structured light patterns, which can be generated by multi-beam interference, projection or point-scanning. ${ }^{3-5}$ This structured pattern is then superimposed on the specimen's fine structures to form the Moiré fringes. By shifting the structured pattern laterally and rotating it in steps on the specimen, several raw images with such Moiré fringes are sequentially recorded, to extend the detectable spectra of microscope in various directions. Following a postprocess with specialized algorithms, the extended spectral bands in each direction are extracted individually and shifted to their true spectral positions, to reconstruct a wide-field image with a lateral resolution increased by approximately twofold. Unlike other super-resolution techniques such as STED, PALM and STORM, SIM provides resolution enhancement at a relatively lower illumination dose and faster acquisition speed. Another benefit of structured illumination is the widespread availability of dyes and fluorescent proteins for labeling specimens and the ease of conducting multicolor imaging.

The main concept of SIM was proposed in 1999 by Heintzmann ${ }^{6}$ and followed by Gustafsson ${ }^{7}$ in 2000. Although the principle of SIM is straightforward, the image reconstruction algorithm in the post-process is complex. ${ }^{8-10}$ The reconstruction programming details in SIM have not been publicly released up to date, making it difficult to conduct the experiment. To give a comprehensive insight and guide the programming, in this work we analyze the whole process of lateral image reconstruction in SIM, including pattern generation, spectra encoding, parameter estimation, spectra decoding, image reconstruction and the analysis of artifacts sources. Since the initial phase of pattern acts as a crucial factor in image reconstruction, a comparison between different methods of pattern phase measurement is carried out, revealing that the auto-correlation method achieves highest performance under a realistic signal-to-noise ratio (SNR).

\section{PSF of Microscope and Image Blur}

The fluorescence light emanating from a fixed point on specimen will become diffracted at the edge of the objective aperture, forming a diffraction pattern with a ring disk in the image plane. This disk is usually referred to as the Airy disk or the point spread function (PSF). As the specimen consists of numerous points, the microscopic image of a specimen can never perfectly represent the real structure of its own, resulting in a blurred image with decreased spatial resolution.

The PSF of a microscope can be defined either theoretically using a scalar diffraction model or experimentally using a sub-resolution point-like source, such as a quantum dot and fluorescent bead. ${ }^{11}$ Since theoretical model allows the numerical calculation of PSF in various systems and conditions, in this work we modeled the PSF of a conventional microscope by using the scalar diffraction approach. ${ }^{11-13}$

According to the Kirchhoff-Fraunhofer approximation, the near-focus three dimensional (3D) PSF in a wide field imaging system can be written as follows: ${ }^{11}$

$$
h\left(x, y, z ; \lambda_{\text {em }}\right)=\operatorname{FT}\left\{P\left(k_{x}, k_{y}, z ; \lambda_{\text {em }}\right)\right\},
$$

where $\left(x, y, z ; \lambda_{\mathrm{em}}\right)$ denotes the distance coordinate on the detector plane at the wavelength $\lambda_{\mathrm{em}}$ of emission light; $\left(k_{x}, k_{y}\right)$ indicates the spatial frequency coordinate on the $z$-stack planes near the exit pupil $(z=0)$ of microscope. $P\left(k_{x}, k_{y}, z ; \lambda_{\text {em }}\right)$ represents the two-dimensional (2D) exit pupil function of the objective (at each defocus $z$ ). FT is the $2 \mathrm{D}$ Fourier transform operator. $h\left(x, y, z ; \lambda_{\mathrm{em}}\right)$ is the PSF in the fluorescence emission path.

The method we used to calculate the 3D PSF of microscope can be found in literature. ${ }^{11}$ In our simulation, the emission wavelength of fluorescence is set to be $515 \mathrm{~nm}$; the numerical aperture of objective $\mathrm{NA}_{\mathrm{obj}}$ is 0.4 ; the refractive index $n_{i}$ and the object's focal length $f$ are 1.525 and $8 \mathrm{~mm}$, respectively; the pixel size of CCD is $8 \mu \mathrm{m}$ and the effective pixel numbers are $1000 \times 1000$, corresponding to the same amount of sampling points in the sample plane; the size of each sample point is identified as $0.4 \mu \mathrm{m}$. The magnification of objective is set as $[20 \times]$, so that a light spot with the lateral size of $8 \mu \mathrm{m}$ which is corresponding to a sample point will be formed on CCD provided this objective is an 


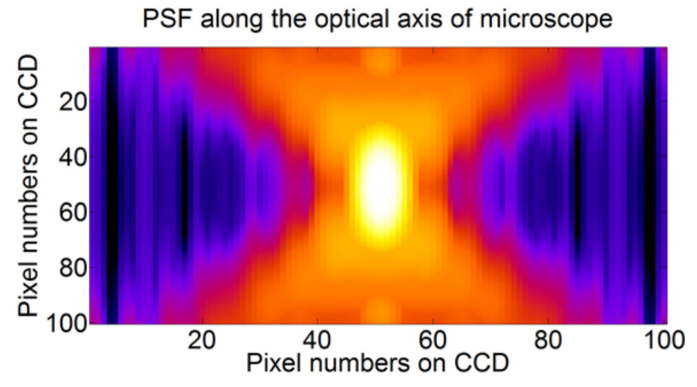

(a)

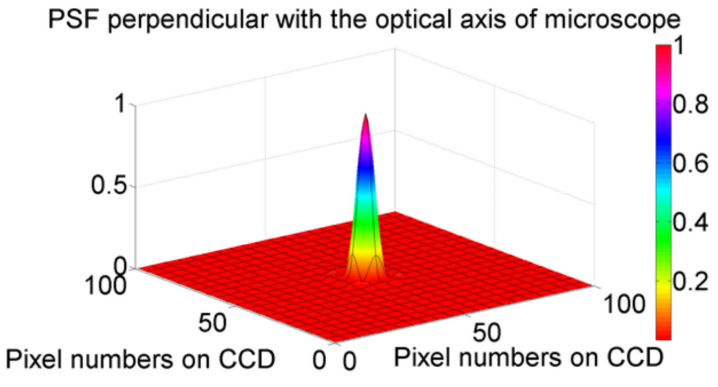

(b)

Fig. 1. PSF of a conventional microscope (a) along the optical axis and (b) across the focal plane of objective.

ideal diffractive-free lens, happening to occupy a single pixel on CCD. However, in the diffractionlimited optical system, the PSF function is extended along the optical axis and across the focal plane of objective as shown in Fig. 1, blurring the ideal light spot.

The blurred image, which restrains the spatial resolution in a diffraction-limited regime, is calculated by convoluting the lateral PSF profile in Fig. 1(b) with the original image, as shown in Fig. 2.

\section{Basic Setup of SIM}

To bypass diffraction limitation and achieve superresolution image, a basic setup of a 2D SIM system using two-beam interference to generate structured patterns is shown in Fig. 3. The SIM system is usually custom-built, for the sake of flexible adjustments. $^{14}$

A coherent laser is normally used as the light source for fluorescence excitation in SIM, if the maximum spatial resolution are wanted; otherwise, a LED light source can be used in place of the laser. The polarizer and the half wave plate placed right behind the laser control the incident beam's polarization, so as to satisfy the polarization requirement of spatial light modulator (SLM). The laser beam is focused by lens L1 through a pinhole $(\mathrm{Ph})$ and collimated with lens L2 to fill the modulation area of a SLM, where a computer-generated digital grating pattern is displayed. The diffracted orders from SLM are focused by lens L3, while the ring filter is used to get rid of the zero-order and lenses L4 and L5 are used for relaying the converged \pm 1 diffraction orders to the back focal plane of the objective, which can be mounted on a motorized $z$-stage. The $\lambda / 2$ plate before L4 is set in a rotating mount and used for rotating the polarization of the input beam so that the two first-orders from SLM are always azimuthally polarized to maximize the contrast of the interference fringes on the specimen. One crucial factor for system adjustment is that both the illumination pattern and specimen structure should simultaneously project their images with best image

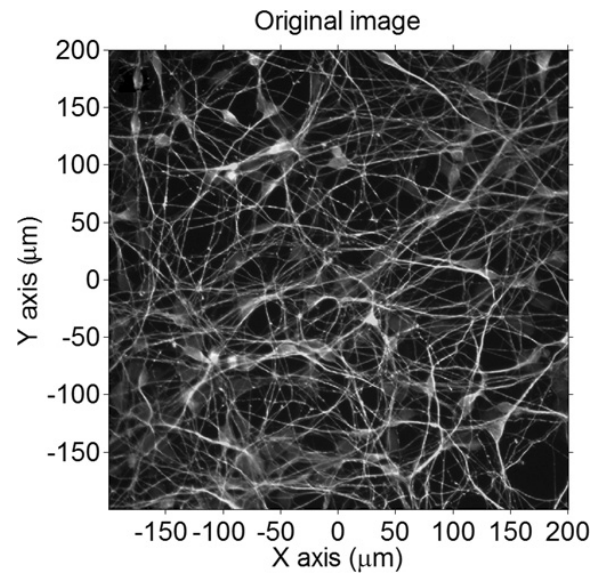

(a)

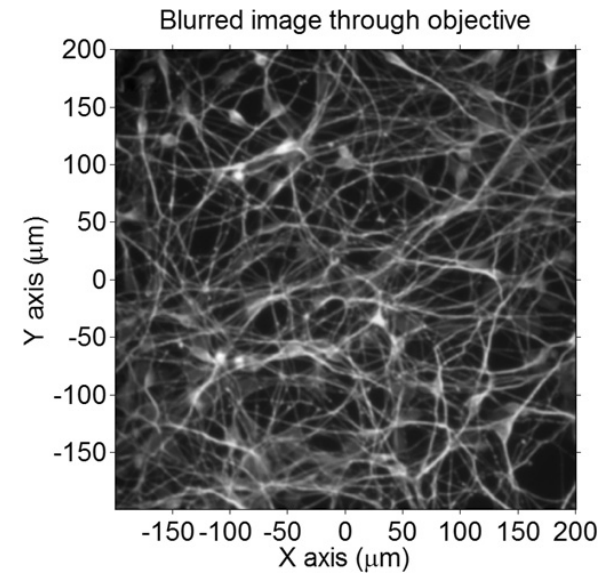

(b)

Fig. 2. Image blurred by the PSF of a conventional microscope. 


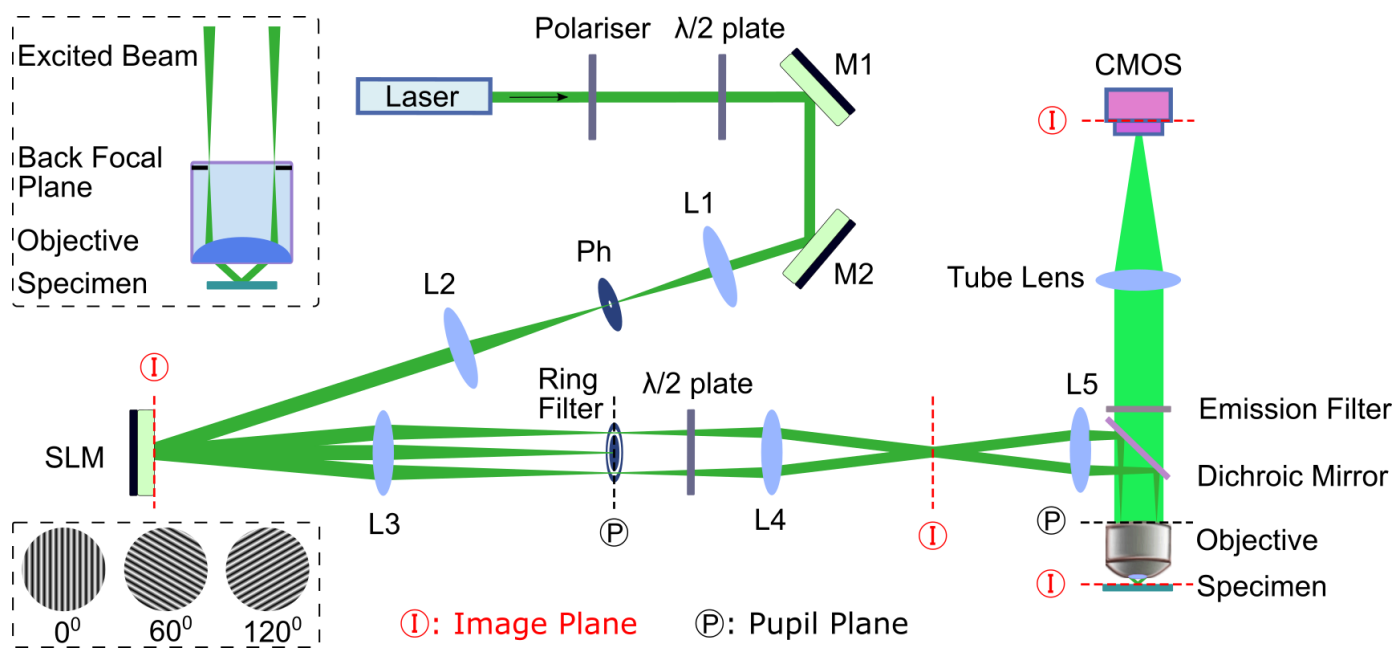

Fig. 3. Schematic setup of a $2 \mathrm{D}$ structure illumination microscope by two beam interference.

contrast on the CMOS sensor through the tube lens. For this purpose, the two exited beams should be adjusted to be parallel before passing through the objective. In the meantime, the two beams' interference plane on specimen should be right in the image plane of SLM's surface, which can be performed by focusing the two exited beams on the back focal plane of objective.

\section{Spectrum Encoding by Structured Illumination}

The moiré fringe is formed when two transparent structured patterns are overlaid together as shown in Fig. 4(a). $d, d_{0}$ and $d_{m}$ are the spatial periods of the specimen, the illumination pattern and the moiré fringes, respectively; $k, k_{0}$ and $k_{m}$ are their corresponding spatial frequencies in Fourier domain. The wave-vector of moiré fringe $\mathbf{k}_{m}$ can be deduced by the superposition principle between $\mathbf{k}_{0}$ and $\mathbf{k}$, as shown in Fig. 4(b). In simplest case, when the two patterns' orientations are parallel $(\alpha=0$ in Fig. 4 , the spatial frequency of moiré fringe $k_{m}$ achieves the minimum which is equal to the difference of two sinusoidal patterns' spatial frequencies. In real experiment, the specimen is composed by fine structures with varied orientation and spacing, leading to a widely distributed $\mathbf{k}$ and $\mathbf{k}_{m}$ in Fourier domain.

The highest frequency of illumination pattern $k_{0}$ in Fig. 4(b) is slightly less than the objective's cutoff frequency $k_{c}$. This is true for the conventional linear SIM in which total internal reflection fluorescence (TIRF) objective is not used and the Stokes shift of emission fluorescence is ignored. In this case, the illumination light and emission light share the same optical path of objective, so that the objective cannot generate an illumination pattern with higher spatial frequency than the highest

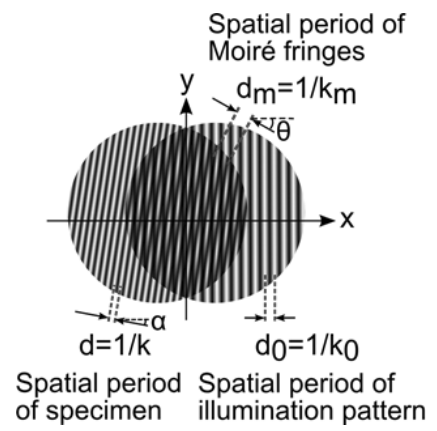

(a)

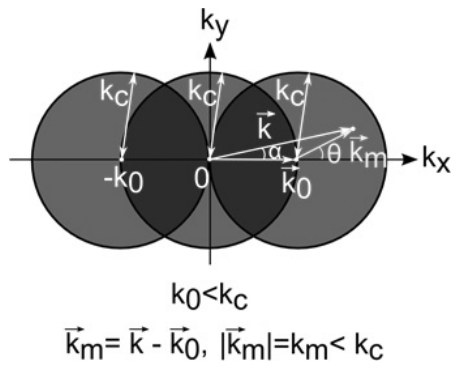

(b)

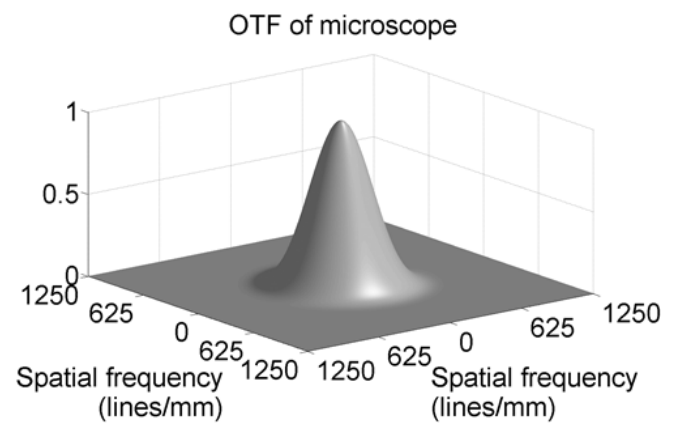

(c)

Fig. 4. Illustration of frequency encoding and decoding in SIM. (a) The moiré fringes generated by structured illumination; (b) the spectral extension in Fourier domain along one orientation and (c) 2D OTF profile of a conventional microscope. 
detectable frequency of microscope in such a linear process. By applying Fourier transformation to one raw image in SIM, at least three overlapped spectral bands will be obtained. The spectral band obtained through the support region of optical transfer function (OTF), as in the conventional microscope, is represented by the center circle in Fig. 4(b). Besides that, due to the moiré effect in SIM, some high frequencies originally outside the OTF's support region are encoded into low frequency circle bands. However, the three circles in Fig. 4(b) are initially spectrally overlapped and fused together before spectral decoding (i.e., spectral separation and recombination). These overlapped spectral circles have to be separated using algorithm and then shifted to their right spectral position according to the location of $\mathbf{k}_{0}$, to extend the detectable spectra as shown in Fig. 4(b). The support region of OTF defines which spatial frequencies the microscope can detect, outside the cut-off frequency the frequency response of OTF is zero. Thus, efforts to extend the detectable spectrum of a microscope, are equivalent to finding a way to obtain the information from outside of the support region of OTF, so as to enhance the image resolution.

The OTF itself describes how strongly, and with what phase shift, the spatial frequency $k$ of the specimen is transferred into the detectable signal. The 2D profile of OTF of microscope, which is the Fourier transform of the corresponding PSF in Fig. 1(b), is drawn in Fig. 4(c) to illustrate the transfer strength of each OTF support region shown in Fig. 4(b). Different from the 3D OTF $\left(k_{x}, k_{y}, k_{z}\right)$ which has a "missing cone" of information near the optical axis of $k_{z},{ }^{8}$ 2D OTF displayed the normalized frequency response of microscope in $2 \mathrm{D}$ spectral domain $\left(k_{x}, k_{y}\right)$, resulting in no display of "missing cone". The vertical axis of $2 \mathrm{D}$ OTF is corresponding to the strength of frequency response which is not usually displayed in a $3 \mathrm{D}$ OTF profile in literatures. The cut-off frequency $k_{c}$ of OTF in Fig. 4(c) is measured to be 750 lines $/ \mathrm{mm}$.

Considering the encoding process by structured illumination, the one-dimensional (1D) sinusoidal structured illumination on the specimen can be expressed as ${ }^{15}$ :

$$
I_{\text {illu }}(x)=I_{0}\left[1+c \cos \left(2 \pi k_{0} x+\varphi\right)\right],
$$

where $k_{0}$ and $\varphi$ denote the spatial frequency and the initial phase of the sinusoidal fringe illumination pattern, respectively. $I_{0}$ and $c$ are both constants called the mean intensity and modulation contrast, respectively. By applying Fourier transform, Eq. (2) can be expressed in frequency space as below by using Euler's formula:

$\tilde{I}_{\text {illu }}(k)=I_{0}\left[\delta(k)+\frac{c}{2} e^{i \varphi} \delta\left(k-k_{0}\right)+\frac{c}{2} e^{-i \varphi} \delta\left(k+k_{0}\right)\right]$,

where tildes $(\sim)$ indicates the Fourier transform of the corresponding real-space quantities. The intensity of emitted fluorescence excited from the specimen before passing through objective is $I_{\text {emi }}(x)=F(x) E(x)=F(x) I_{\text {illu }}(x)$. Here, $E(x)$ is the emission-rate per fluorophore which is proportional to $I_{\text {illu }}(x)$ for the linear SIM, $F(x)$ is the density of fluorophores on the specimen. Since $F$ ( $x)$ indicates the structure of the sample $S(x)$, the output power spectrum on the detector after the excited fluorescence have passed through the objective is:

$$
\begin{aligned}
\tilde{I}_{\mathrm{det}}(k)= & \tilde{I}_{\mathrm{emi}}(k) \cdot \mathrm{OTF}(k) \\
= & {\left[\tilde{F}(k) * \tilde{I}_{\mathrm{illu}}(k)\right] \cdot \operatorname{OTF}(k) } \\
= & {\left[\tilde{S}(k) * \tilde{I}_{\mathrm{illu}}(k)\right] \cdot \operatorname{OTF}(k) } \\
= & I_{0}\left[\tilde{S}(k)+\frac{c}{2} e^{i \varphi} \tilde{S}\left(k-k_{0}\right)\right. \\
& \left.+\frac{c}{2} e^{-i \varphi} \tilde{S}\left(k+k_{0}\right)\right] \cdot \operatorname{OTF}(k),
\end{aligned}
$$

where $*$ indicates the convolution operator. In this way, some spatial frequencies of specimen $k$ higher than the cut-off frequency $k_{c}$ of objective are encoded into a low-frequency circular band with the radius of $\left|\mathbf{k}_{m}\right|$, which is inside the radius of $k_{c}$ of microscope. In other words, some original undetectable band is now transited to the support region of $2 \mathrm{D} \mathrm{OTF}$ and become detectable.

In regards to the simulation presented in this work, three images are captured for each orientation of the grating. By step-rotating the orientation of grating by $60^{\circ}$ as shown in the inset of Fig. 3, nine raw images in total are obtained in three different grating orientations and then are separated and recombined together to produce a final super-resolution image, which will be discussed in Sec. 5 .

In our work, two Gaussian beams with $0.5 \mathrm{~mm}$ diameter interference with each other at an included angle of $45^{\circ}$, making the spatial frequency of grating pattern a little less than the cut-off frequency $k_{c}$ (750 lines $\left./ \mathrm{mm}\right)$ and still in the support region of $\mathrm{OTF}$. 


\section{Spectrum Decoding for Image Reconstruction}

To obtain the super-resolution fluorescence image in SIM, the high frequencies of specimen which are encoded through structured illumination in Eq. (4), must be decoded in the data post-processing. For this purpose, structured patterns having different initial phases $\varphi_{n}$ are sequentially illuminated on specimen to produce three independent linear equations in a matrix form, aiming to resolve the sample information $S(x)$ provided that $a$ and OTF $(k)$ are pre-known ${ }^{15}$ :

$$
\begin{aligned}
& \left(\begin{array}{l}
\tilde{I}_{\text {det_1 }}(k) \\
\tilde{I}_{\text {det_2 }}(k) \\
\tilde{I}_{\text {det_3 }}(k)
\end{array}\right) \\
& =I_{0}\left(\begin{array}{ll}
1 \frac{c}{2} e^{i \varphi_{1}} & \frac{c}{2} e^{-i \varphi_{1}} \\
1 \frac{c}{2} e^{i \varphi_{2}} & \frac{c}{2} e^{-i \varphi_{2}} \\
1 \frac{c}{2} e^{i \varphi_{3}} & \frac{a}{2} e^{-i \varphi_{3}}
\end{array}\right)\left(\begin{array}{l}
\tilde{S}_{0}(k) \\
\tilde{S}_{+1}\left(k-k_{0}\right) \\
\tilde{S}_{-1}\left(k+k_{0}\right)
\end{array}\right) \operatorname{OTF}(k) \\
& =I_{0} \Phi\left(\begin{array}{l}
\tilde{S}_{0}(k) \\
\tilde{S}_{+1}\left(k-k_{0}\right) \\
\tilde{S}_{-1}\left(k+k_{0}\right)
\end{array}\right) \operatorname{OTF}(k),
\end{aligned}
$$

where $\tilde{I}_{\text {det_n}}(k)$ is the Fourier transform of the $n$th raw image under illumination of the $n$th structure pattern with phase $\varphi_{n}(n=[1,2,3]$ here $)$. The matrix $\Phi$ mixes different spectral component $\tilde{S}_{m}(k-$ $\left.m k_{0}\right)$ into $\tilde{S}(k)=\sum_{m=-M}^{M} \tilde{S}_{m}\left(k-m k_{0}\right)$, where $M$ is the maximum order of the pattern's harmonic frequencies (equal to 1 in linear SIM) and $\Phi$ can be further decomposed into two parts:

$$
\Phi=C \cdot \Psi=\left(\begin{array}{lll}
1 & c / 2 & c / 2 \\
1 & c / 2 & c / 2 \\
1 & c / 2 & c / 2
\end{array}\right) \cdot\left(\begin{array}{lll}
1 & e^{i \varphi_{1}} & e^{-i \varphi_{1}} \\
1 & e^{i \varphi_{2}} & e^{-i \varphi_{2}} \\
1 & e^{i \varphi_{3}} & e^{-i \varphi_{3}}
\end{array}\right)
$$

Each $\tilde{I}_{\text {det_n}}(k)$ in Eq. (6) can be generally expressed as follows, if ignoring the constant $I_{0}$ :

$$
\begin{aligned}
\tilde{I}_{\text {det } \_n}(k) & =\sum_{m=-M}^{M} \tilde{I}_{\text {det } \_n \_m}(k) \\
& =\sum_{m=-M}^{M} c_{m} e^{i m \varphi_{n}} \tilde{S}_{m}\left(k-m k_{0}\right) \operatorname{OTF}(k) .
\end{aligned}
$$

Both the modulation contrast $c$ and $\operatorname{OTF}(k)$ should be measured in advance to recover the sample structure $\tilde{S}(k)$, which will be discussed in Sec. 6. If $\operatorname{OTF}(k)$ is not measured in advance, one can only obtain $\tilde{E}(k)$ instead of $\tilde{S}(k)$, but still extends the original observable spectrum $\tilde{S}_{0}(k) \operatorname{OTF}(k)$ as shown below if combing different $\tilde{E}_{m}\left(k-m k_{0}\right)$ into $\tilde{E}(k)$ in Fourier domain:

$$
\begin{aligned}
\left(\begin{array}{l}
\tilde{E}_{0}(k) \\
\tilde{E}_{+1}\left(k-k_{0}\right) \\
\tilde{E}_{-1}\left(k+k_{0}\right)
\end{array}\right) & =\left(\begin{array}{l}
\tilde{S}_{0}(k) \\
\tilde{S}_{+1}\left(k-k_{0}\right) \\
\tilde{S}_{-1}\left(k+k_{0}\right)
\end{array}\right) \operatorname{OTF}(k) \\
& =\Phi^{-1}\left(\begin{array}{c}
\tilde{I}_{\text {det_1 }}(k) \\
\tilde{I}_{\text {det_2 }}(k) \\
\tilde{I}_{\text {det_3 }}(k)
\end{array}\right) / I_{0},
\end{aligned}
$$

where the un-mixing matrix $\Phi^{-1}$ is the inverse matrix of $\Phi$. Since these spectral terms are overlapped initially in Fourier domain, they have to be shifted by $\left(0,+k_{0},-k_{0}\right)$ separately to their true origins, finally assembling an extended spectrum. To further obtain nearly isotropic resolution enhancement, the single orientation sinusoidal fringe pattern should be rotated in three orientations as displayed in Fig. 5(a).

The image reconstructed only by the extended pass bands corresponding to the high frequencies outside the blue circle in Fig. 5(a), is shown in Fig. 5(b). The effect of image edge enhancement reveals the principle of resolution enhancement in SIM. The blurred image in a conventional microscope and the reconstructed image via spectrum extension in a SIM are both displayed in Fig. 6 for comparison. In the small area with dotted circle, the effect of spatial resolution enhancement can be clearly observed. To further improve the image resolution, the real spectral information of specimen $\tilde{S}(k)$ must be calculated through deconvolution after $\tilde{E}(k)$ is obtained, provided OTF is known. Besides the calculation approach which is used to obtain OTF in this work as shown in Fig. 4(c), there are several methods available to experimentally measure OTF by imaging sub-resolution microspheres. ${ }^{12,13}$ After the OTF is obtained, a Weiner filter in spectral domain is usually used in SIM for deconvolution since it can suppress the white noise in raw images ${ }^{16}$, which will be discussed in Sec. 7 .

Using the commercial objective with highest NA and magnification, an increased lateral resolution of $\sim 130 \mathrm{~nm}$ can be obtained in linear SIM, approximately two-fold of conventional microscope. This 


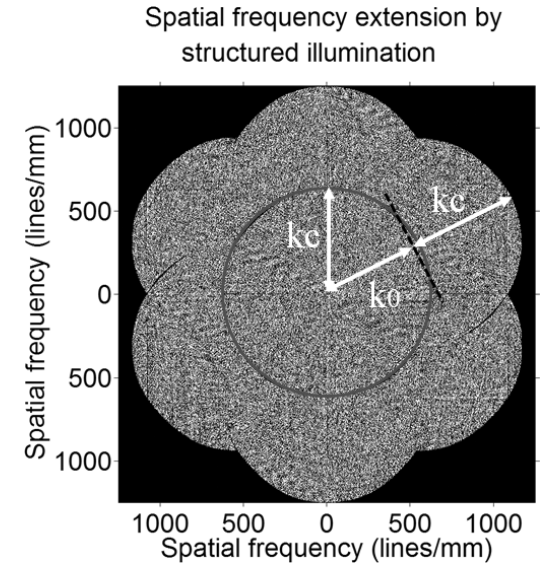

(a)

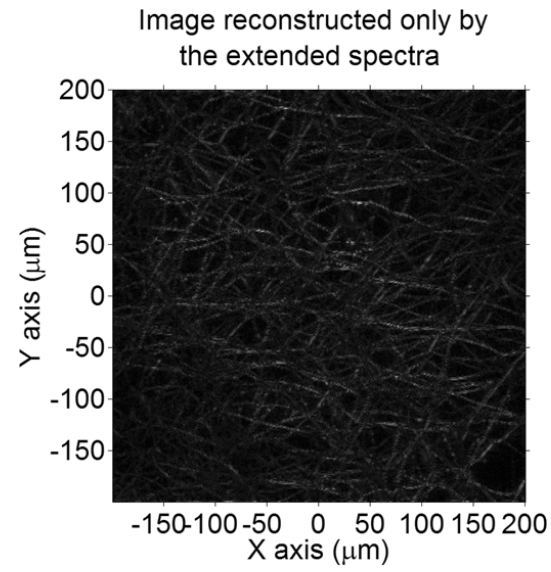

(b)

Fig. 5. Spectrum extension (a) and image reconstruction and (b) in two directions by structured illumination.

resolution can be improved further to sub $100 \mathrm{~nm}$ by using the TIRF scheme, where the two evanescent waves move on the specimen surface opposite with each other, leading to the spatial frequency of interference pattern achieves maximum. The relationship between the number of gratings rotations and the recovered image resolution depends on the grating spatial frequency, as well as the geometrical relationship between the grating lines and prominent specimen features. In general, the final resolution can be enhanced by increasing the number of grating rotations at the cost of acquisition speed and increased photo-bleaching. One main drawback of SIM is the time consuming process (normally 0.1 to $30 \mathrm{~s}$ ) necessary to reconstruct a super-resolution image. ${ }^{9,17-19}$

Nonlinear SIM operates with the same process of linear SIM, but produces higher resolution by generating the harmonic spatial frequencies of the illumination pattern through nonlinear response of fluorescence yield rate. ${ }^{20}$ Considering the simplest case, the nonlinear function $f(I)$ of illumination intensity $I$ can be described by the Taylor series $f(I(x))=a_{1} I(x)+a_{2} I(x)^{2}+a_{3} I(x)^{3}+\cdots$ and the

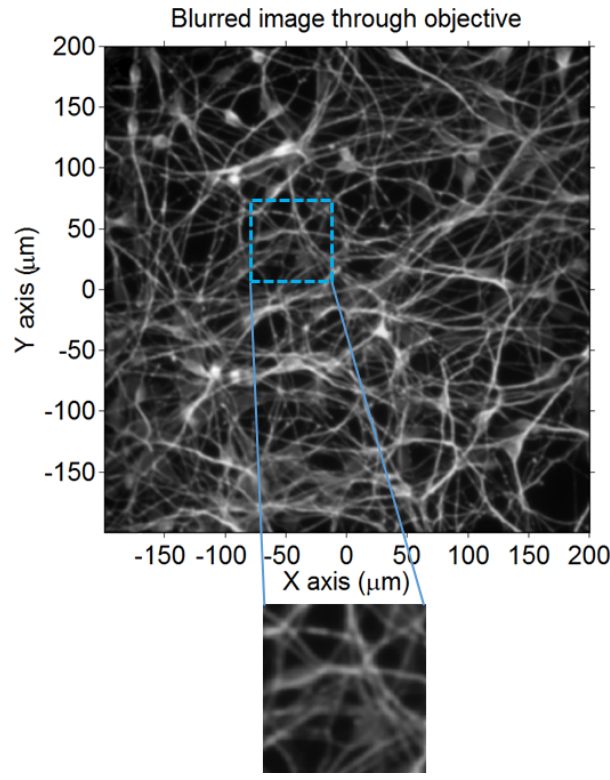

(a)

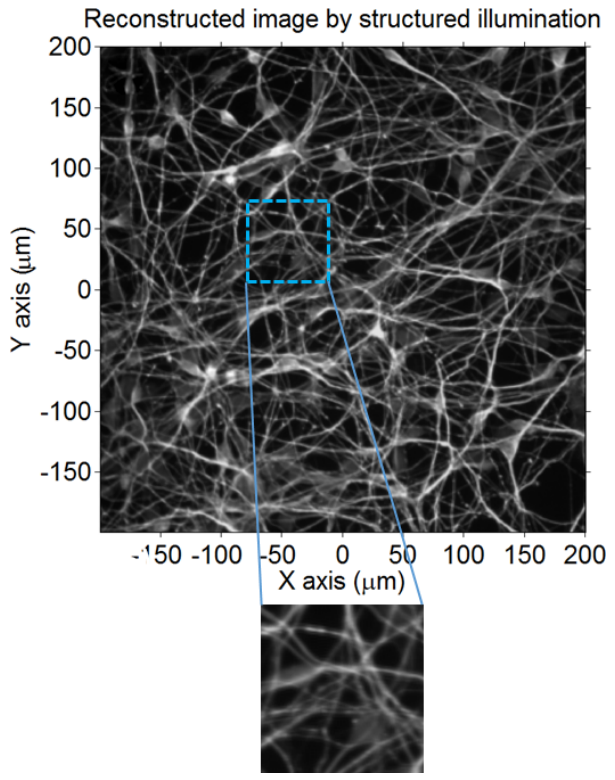

(b)

Fig. 6. Comparison of image resolution in conventional microscope and SIM. (a) Blurred image through objective in a conventional microscope and (b) Reconstruction image by structured illumination. 


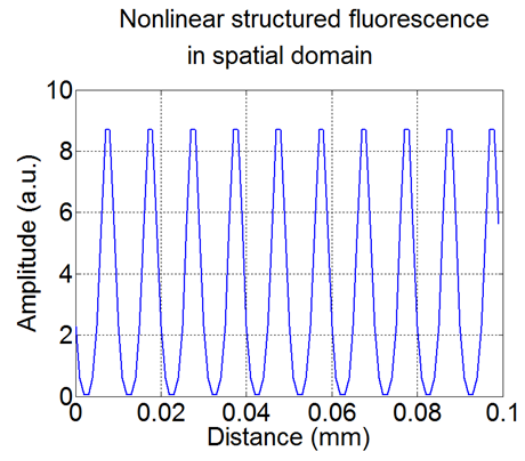

(a)

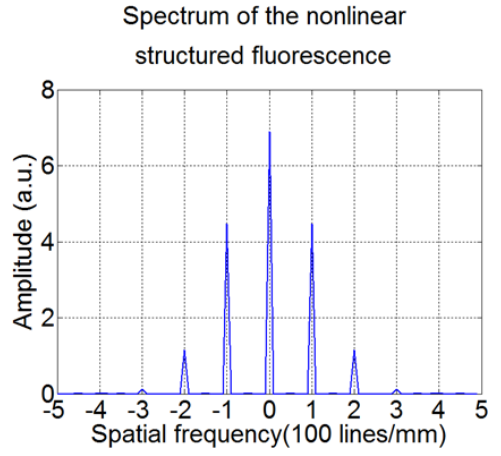

(b)

Fig. 7. Harmonic frequencies deduced by the structured illumination in nonlinear SIM.

normalized structured illumination intensity $I(x)=$ $1+\cos \left(2 \pi k_{0} x\right)$. Since the emission rate $E(x)$ per fluorophore is proportional to $f(I)$, the emission fluorescence intensity of specimen with fluorophore density $F(x)$ is therefore:

$$
\begin{aligned}
I_{\mathrm{emi}}(x)= & F(x) E(x) \\
= & F(x)\left[a_{1}\left(1+\cos \left(2 \pi k_{0} x\right)\right)\right. \\
& +a_{2}\left(1+\cos \left(2 \pi k_{0} x\right)\right)^{2} \\
& \left.+a_{3}\left(1+\cos \left(2 \pi k_{0} x\right)\right)^{3}+\cdots\right] .
\end{aligned}
$$

The $m$ th term in this series is proportional to $I(x)=\left(1+\cos \left(2 \pi k_{0} x\right)\right)^{m}$, which has a decomposed term in the form of $I(x)=\cos \left(2 \pi\left(m k_{0}\right) x\right)$, resulting in the generation of harmonic frequency $m k_{0}$. An example of nonlinear emission function $f(I(x))$ is shown in Fig. $7(\mathrm{a})$, where $a_{1}=1, a_{2}=0.8, a_{3}=$ $0.5, k_{0}=100 \mathrm{~mm}^{-1}(100$ lines $/ \mathrm{mm})$. The corresponding power spectrum with harmonic frequencies $m k_{0}(m= \pm 1, \pm 2, \pm 3)$ is calculated after taking the Fourier transform to $f(I(x))$, as shown in Fig. $7(\mathrm{~b})$. The detectable frequency $\mathbf{k}$ is getting much larger in nonlinear SIM, since $\mathbf{k}_{m}=\mathbf{k}+\mathbf{k}_{0}$ in linear SIM is replaced by $\mathbf{k}_{m}=\mathbf{k}+m \mathbf{k}_{0}$ due to the harmonic frequencies. Although a nonpolynomial nonlinear function has an infinite number of Taylor series terms which permits theoretically unlimited resolution in SIM, the strength of the harmonics declines rapidly with increased orders $m$, leading to the finite resolution of SIM in practice under a realistic SNR.

Beside nonlinear SIM, another type of SIM called plasmonic structured illumination microscopy (PSIM) which can possess higher image resolving power compared with conventional SIM was proposed. ${ }^{21}$ PSIM used surface plasmon interference as an illumination source, without utilizing nonlinear dependence of the fluorescence emission rate on the illumination intensity.

\section{Phase Shift Estimation in Structured Illumination Patterns}

In SIM, the specimen is sequentially illuminated by periodic structured patterns having different pattern positions, which are also called as different initial phases. According to Eq. (8), separating the individual spectral terms $\tilde{S}_{0}(k), \tilde{S}_{+1}\left(k-k_{0}\right)$ and $\tilde{S}_{-1}\left(k+k_{0}\right)$ requires the accurate knowledge of the initial phases $\varphi_{n}$, which can be obtained a priori by trying to synchronize the pattern displayed on SLM with the illumination pattern on specimen, or $a$ posteriori by directly measuring the pattern on specimen. If these initial phases on specimen cannot be precisely controlled experimentally, artifacts will be produced due to incorrect separation of spectral terms in the reconstructed image, which makes the estimation of the initial phases or the phase shifts on specimen a crucial factor in SIM. ${ }^{22,23}$

The initial phase of structured illumination pattern, according to Eq. (2), can be explained as the phase deference between two parallel incident plane waves before passing through the objective to interfere with each other, or further explained as the phase deference of two oblique incident beams at the central point of their overlapped interference region on the sample surface. However in most cases of SIM, the phase or the pattern position at that sample point cannot be measured directly in any intermediate image plane by using the notable method of fringe analysis, because the fine structures of illumination patterns have very low 
contrast since their spatial frequencies are located near the support edge of OTF, making themselves nearly unobservable on the detector especially in a noisy condition. Even in an ideal noise-free condition with observable pattern contrast, the directly measured phase via fringe analysis is probably not the real initial phase, since it mainly depends on how the image detector is placed in respect to the sample plane. The alternative method, in contrast, is to achieve the initial phase through measuring its indirect impact applied to the power spectra of the raw images, resulting in the capability of operating effectively even at the pattern frequency higher than the cut-off frequency of microscope.

There are at least three different methods to measure the initial phases having been proposed up to date. A Fourier-based approach, which is similar with the fringe analysis method and proposed by Shroff, calculates the phase spectrum at the peak frequency of the structured pattern, leading to a high precision estimation of the random initial phases under relatively high SNR. ${ }^{22}$ In detail, the Fourier transform of a raw captured image at the pattern frequency $k_{0}$, is as below according to Eq. (4):

$$
\begin{aligned}
\tilde{I}_{\operatorname{det}}\left(k_{0}\right)= & I_{0}\left[\tilde{S}\left(k_{0}\right)+\frac{c}{2} e^{i \varphi} \tilde{S}(0)+\frac{c}{2} e^{-i \varphi} \tilde{S}\left(2 k_{0}\right)\right] \\
& \times \operatorname{OTF}\left(k_{0}\right) .
\end{aligned}
$$

The initial phase in this equation can be retrieved as below if three pre-conditions are satisfied: (a) the pattern contrast $c$ is sufficiently high (at least $>0.1$ ); (b) the magnitude of OTF at $k_{0}$ should be inside the support of OTF and sufficiently large; (c) the specimen's power spectral at zero frequency $|\tilde{S}(0)|^{2} \gg\left|\tilde{S}\left(k_{0}\right)\right|^{2}+\left|\tilde{S}\left(2 k_{0}\right)\right|^{2}$, which is a reasonable assumption for most natural samples.

$$
\begin{aligned}
\varphi & =\arg \left[I_{0} \frac{c}{2} e^{i \varphi} \tilde{S}(0) \cdot \operatorname{OTF}\left(k_{0}\right)\right] \\
& \approx \arg \left[\tilde{I}_{\operatorname{det}}\left(k_{0}\right)\right],
\end{aligned}
$$

where arg is the abbreviation of complex argument. This equation works when both $\tilde{S}(0)$ and $\operatorname{OTF}\left(k_{0}\right)$ are real-valued constants, which are the case if the real-valued samples with incoherent fluorescence emission and the optical lens with symmetrical PSF are used. Two limitations exist for this method. First, when the white noise of image is strong or the pattern has fine structure with high frequency which is near the edge of OTF, the pattern contrast will become much lower, making the approximation in Eq. (11) not true so that this method will fail. Second, due to the Stokes shift of emission fluorescence or the application of TIRF, the spatial frequency of patterns will be getting higher even outside the detection regime of OTF, letting this Fourier-based method fail since $\operatorname{OTF}\left(k_{0}\right)$ will be zero outside the detection regime.

To overcome the limitations above, an iterative optimization method calculating the crosscorrelation between separated spectral components was proposed, which has the ability to estimate the initial phases of patterns from noisy images or images with fine structured patterns. ${ }^{23}$ The principle of this method is as followed: if the spectral terms in one raw image are separated perfectly due to the accurate initial phase estimation, the crosscorrelation tensor between an un-shifted terms $\tilde{E}_{i}(k)$ and a shifted (but un-overlapped with $\tilde{E}_{i}(k)$ ) terms $\tilde{E}_{j}\left(k-l k_{0}\right)$ should be getting lowest at frequency $k=0$. In short, the accurate initial phase is responsible to the perfect separation of spectral terms, which leads to the minimum cross-correlation values. To achieve the optimal phase values, an optimization process was used, to minimize a cost function $g(\Phi)$ while iteratively optimizing the inverse mixing matrix $\Phi^{-1} \cdot g(\Phi)$ is equal to the superimposition of the squared rooted cross-correlation tensors $\xi$ between separated Fourier terms:

$$
\begin{aligned}
g(\Phi) & =\sum_{j+-i \neq 0}|\xi|^{1 / 2} \\
& =\sum_{j+l-i \neq 0}\left|\left[\tilde{E}_{i}(k) \otimes \tilde{E}_{j}\left(k-l k_{0}\right)\right]\right|_{k=0}^{1 / 2},
\end{aligned}
$$

where $i$ and $j$ are the order numbers of spectral terms (which are $[0,1,-1]$ for linear SIM) for the $n$th raw image, $l=1$ to $L$ are the integer numbers while $L k_{0}$ the highest shift being still inside the OTF support region, $\otimes$ is the operator of cross-correlation, and the terms $\tilde{E}_{i}(k)$ and $\tilde{E}_{j}\left(k-l k_{0}\right)$ can be calculated from $\tilde{E}_{m}\left(k-m k_{0}\right)=\Phi_{n \_m}^{-1} I_{\text {det } \_n \_m}(k) / I_{0}$ according to Eq. (7). $\xi$ is calculated between the $i$ th separated but un-shifted term at frequency $k=0$ (i.e., the central point in this spectral term) and the $j$ th separated term shifted by frequencies $l k_{0}$, in the condition of $i \neq j+l$ which makes the two terms un-overlapped in Fourier domain. While $\Phi$ is slightly changed for optimization each time, $\tilde{E}_{i}(k)$ 
has to be re-calculated again for cross-correlation, which slows down the whole process. To speed up the calculation, the cost function $g(\Phi)$ can be adjusted as shown below:

$$
\begin{aligned}
g(\Phi) & =\sum_{\substack{j+l-i \neq 0 \\
k=0}}\left|\Phi_{n i}^{-1} \tilde{I}_{\text {det_n_i }}(k) \otimes \Phi_{n_{-} j}^{-1} \tilde{I}_{\text {det_n_j }}\left(k-l k_{0}\right)\right|^{1 / 2} \\
& =\sum_{\substack{j+l-i \neq 0 \\
k=0}}\left|\Phi^{-1} \cdot \gamma \cdot\left(\Phi^{-1}\right)^{*}\right|^{1 / 2},
\end{aligned}
$$

where $\gamma=\left[\tilde{I}_{\operatorname{det}_{\tilde{I}} n_{-} i}(k) \otimes \tilde{I}_{\text {det_n } n_{-j}}\left(k-l k_{0}\right)\right]_{k=0}=\sum_{i, j}$ $\times\left[\tilde{I}_{\text {det_n } \_i}(k) \cdot\left(\tilde{\tilde{I}}_{\text {det_n} \_j}\left(k-l k_{0}\right)\right)^{*}\right]_{k=0}$, and the symbol ${ }^{*}$ represents the conjugate operation. The performance of this method can be further improved by using weighted cross-correlation tensors $\xi$, to maximize the ratio of information correlation values against noise correlation values. ${ }^{23}$ Compared with Eq. (12), while the values inside $\Phi$ are changed for optimization here, only the multiplications instead of the cross-correlations need to be re-calculated, thus saving the calculation time. In this way, this cross-correlation based method is relatively fast, but its iterative nature inevitably leads to longer consuming time than the noniterative phaseof-peak approach proposed by Schroff. Furthermore, it potentially could yield wrong phases if iteration is getting stuck in a local minimization process.

To keep the advantages of the cross-correlative approach while catching up with the calculation speed of the phase-of-peak method, an alternative auto-correlation method which uses weighted autocorrelations of individual raw Fourier images was proposed. ${ }^{24}$ This method correlates shifted sample components at the pattern peak frequency $k_{0}$ inside the $i$ th Fourier image which is defined as the Fourier transform of the $i$ th captured raw image in real space under illumination by one structured pattern. According to Eq. (7), the Fourier image has the expression of $\tilde{I}_{\text {det_n }}(k)=\sum_{m=-M}^{M} \tilde{I}_{\text {det_n_m }}(k)=$ $\sum_{m=-M}^{M} c_{m} e^{i m \varphi_{n}} \tilde{S}_{m}\left(k-m k_{0}\right) \operatorname{OTF}(k)$, where $M$ is the order number of harmonic frequencies in the structured pattern. Thus, the auto-correlation tensor of $\tilde{I}_{\text {det_n } n}(k)$ is calculated as:

$$
\zeta_{n}=\left[\tilde{I}_{\text {det_n }} \otimes \tilde{I}_{\text {det_n}}\right]_{k=k 0}
$$

$$
\begin{aligned}
& =\sum_{m=-M}^{M} \sum_{m^{\prime}=-M}^{M}\left[\tilde{I}_{\mathrm{det} \_n \_m} \otimes \tilde{I}_{\mathrm{det} \_n \_m^{\prime}}\right]_{k=k 0} \\
& =\sum_{m=-M}^{M} \sum_{m^{\prime}=-M}^{M} \exp \left[i\left(m-m^{\prime}\right) \varphi_{n}\right]\left\{c_{m} \cdot c_{m^{\prime}} \cdot \kappa_{m, m^{\prime}}\right\},
\end{aligned}
$$

where $c_{m}$ and $c_{m^{\prime}}$ are the contrasts of structured illumination pattern for the $m$ th and $m^{\prime}$ th components which are the matrix elements inside $C$ in Eq. (6), and the 2D integral term $\kappa_{m, m^{\prime}}=\int \tilde{S}\left(k-m k_{0}\right)$ $\tilde{S}^{*}\left(k-\left(m^{\prime}-1\right) k_{0}\right)|\mathrm{OTF}(k)|^{2}\left|\mathrm{OTF}\left(k+k_{0}\right)\right|^{2} d k$. In essential, this noniterative auto-correlation based method sums up the cross-correlated values at frequency $k_{0}$ between the pairs of adjacent Fourier components (i.e., $m^{\prime}=m+1$ ) which have a relative distance of $k_{0}$ in spectral domain, while ignoring the small cross-correlated values between un-adjacent components (i.e., $m^{\prime} \neq m+1$ ). The cross-correlated values at $m^{\prime}=m+1$ are much larger than the ignored ones at $m^{\prime} \neq m+1$, because the adjacent components have an overlapped frequencies zone while the un-adjacent ones have not. Moreover, the frequency $k_{0}$ at which the correlations are calculated is chosen to be equal to the relative distance between the adjacent components, which maximizes the correlated values at $m^{\prime}=m+1$ and further highlights their contributions in Eq. (14), finally leading to $\zeta_{n} \approx$ $\exp \left(-i \varphi_{n}\right) \sum_{m=-M}^{M-1}\left\{c_{m} \cdot c_{m+1} \cdot \kappa_{m, m+1}\right\}$. Thus, the initial phase can be expressed as:

$$
\varphi_{n} \approx-\arg \left\{\xi_{n}\right\}_{k=k_{0}}=-\arg \left\{\left[\tilde{I}_{\text {det_nn }} \otimes \tilde{I}_{\text {det_n}}\right]_{k=k 0}\right\} .
$$

It is worth noting that the Fourier image $\tilde{I}_{\text {det_n }}$ is better to be multiplied with the complex conjugated $\operatorname{OTF}^{*}(k)$ in advance, to weigh itself so as to suppress the white noise. In comparison, the three methods discussed above for pattern phase retrieval are summarized in Table $1 .^{22-24}$ The two correlation methods can measure the pattern phase even when the pattern's spatial frequency is higher than the cut-off frequency, because they essentially achieve the phase values by measuring the pattern phase's impact applied in the spectra of raw images instead of directly observing the pattern shape which has very low contrast. An optional way in SIM to conduct the image reconstruction while not knowing the initial phases, is to precisely control the relative phase shifts between the successively translated structured patterns in place of measuring their 
Table 1. Comparison of three deferent methods to retrieve the initial phase of structured pattern. ${ }^{22-24}$

\begin{tabular}{lccc}
\hline \multicolumn{1}{c}{ Methods } & Phase at the pattern frequency & Cross-correlation & Auto-correlation \\
\hline Algorithm type & Single step & Iteration & Single step \\
Process space & Fourier space & Fourier space & Fourier space \\
Applicable structured pattern & Only coarse & Coarse or fine & Coarse or fine \\
Acquisition speed & ++ & + & ++ \\
Precision under realistic SNR & + & + & ++ \\
( $>10$ photons per pixel) & $<\lambda / 100$ for coarse pattern & $<\lambda / 100$ & $<\lambda / 500$ \\
Robustness under realistic SNR & + & + & ++ \\
\hline
\end{tabular}

initial phases, provided this controlling is accurate enough such as in a system with small aberrations. The principle behind is as below: no matter what values the three initial phases are in Eq. (5), as long as they are originally equidistant (such as $0^{\circ}, 120^{\circ}, 240^{\circ}$ ) and added simultaneously by a same phase delay, the reconstructed image quality wouldnot be changed. ${ }^{22}$

The "standard" reconstruction algorithms discussed above relies on precise knowledge of the illumination pattern (phase, grating period, etc.) and are sensitive to artifacts in the case of nonideal experimental conditions. For example, the inevitable experimental imprecision and sample inhomogeneity which lead to an undesirable distortion of the illumination patterns will produce artifacts and lower the reconstruction quality. To overcome this problem and make SIM more robust, a novel statistical approach referred to as blind-SIM which essentially does not require a priori knowledge of the illumination pattern was developed. ${ }^{25}$

Blind-SIM is based on the blind deconvolution theory aiming to solve the inverse problem in image reconstruction, which can estimate the parameters (sample structure) of a linear time-invariant imaging system by measuring the output signal (fluorescence emission) even though the input signal (illumination patterns) is unknown a priori. Besides the sample structure, the unknown illumination patterns itself can also be recovered simultaneously in blind-SIM by adding some reasonable constrains or assumptions, no matter the patterns are periodic, nonperiodic or even speckled. ${ }^{25}$ Blind-SIM adopted the maximum likelihood estimation from parameter estimation methods in statistics, which is also one of the iterative methods typically used in blind deconvolution, to perform the reconstruction process whereby each iteration improves the estimation of the system parameters and the input signal. Recently, the performance of the blind-SIM algorithm has been further improved by using partial a priori knowledge of the illumination pattern. This socalled filtered blind-SIM has enabled to reconstruct the $2 \mathrm{D}$ sample excited by strongly distorted light fringes while improving the previous blind-SIM algorithm to make it efficient in dealing with nondistorted periodic patterns. ${ }^{26}$ To achieve optical sectioning reconstruction in thick samples, another novel implementation of blind-SIM, called thick slice blind-SIM, has been developed to remove the out-of-focus light contribution out of data acquired in a chosen plane. ${ }^{27}$ These kinds of blind-SIMs will widen the applications of SIM, especially if their iteration speeds can run faster.

\section{Parameters Acquisition and Image Reconstruction Procedure}

To obtain the initial phase by using one of the three methods discussed in Sec. 6, as shown in Eqs. (10), (12) and (14), some other important parameters have to be pre-known. These parameters include the pattern frequency $k_{0}$ (as well as its vector direction) and the pattern contrast $c_{m}$, both of which can be achieved in Fourier space as following. ${ }^{8}$

The pattern frequency $k_{0}$ can be determined by a cross-correlation optimization process. Considering of the two items $M 1=\tilde{I}_{\text {det_n_m }}\left(k+m k_{0}\right)$ $\operatorname{OTF}(k)$ and $M 2=\tilde{I}_{\text {det_n_n}}(k) \mathrm{OTF}\left(k+m k_{0}\right)$, both of them have the same expression of $\tilde{S}(k) \mathrm{OTF}(k)$ $\mathrm{OTF}\left(k+m k_{0}\right)$, except for a fixed factor $c_{m} e^{i m \varphi_{n}}$ while ignoring the noises, resulting in $M 1=$ $c_{m} e^{i m \varphi_{n}} \cdot M 2$. The principle behind this optimization method is based on the fact that $M 1$ and $M 2$ are overlapped in frequency domain (when $m=+1$ or -1 in linear SIM) if using the correct pattern frequency $k_{0}$, at which their cross-correlation values will achieve maximum. Before this cross-correlation, the individual values of $M 1$ and $M 2$ need to be calculated as in the following process. $\mathrm{OTF}(k)$ and 
$\mathrm{OTF}\left(k+m k_{0}\right)$ can be determined as followed by recording the fluorescence signal from a fluorescent bead in sample plane under structured illumination. The $n$th Fourier image of this fluorescent bead consists of $m$ separated components and the $m$ th one is $\tilde{I}_{\text {det_n_m } m}(k)=c_{m} e^{i m \varphi_{n}} \tilde{S}\left(k-m k_{0}\right) \operatorname{OTF}(k)$ as in Eq. (7). These components can be resolved respectively from Eq. (5), provided several raw images with pre-known relative phase shifts have been recorded along the corresponding direction. This is possible to achieve by using a precisely-controlled SLM in a setup with small aberrations. And the pattern contrast $c_{m}$ is originally a constant and can be measured straightforward from the intensity variation of the fluorescence bead. If the light emission from a fluorescent bead is approximately regarded as a delta function, its power spectrum $\tilde{S}(k)$ is approximately a constant at any frequency, which means $\tilde{S}\left(k-m k_{0}\right) \approx \tilde{S}\left(k+m k_{0}\right)$. Thus, if $\tilde{I}_{\text {det_n_ }+m}(k)$ is divided by $\tilde{I}_{\text {det_n_-m }}(k)$ (for example, $m=1$ ), the initial phase $e^{i \varphi_{n}}$ which is solely related to the random location of the bead and irrelevant to our measurement, can be retrieved and removed from $\tilde{I}_{\text {det_n_m}}$, leading to a modified $\tilde{I}_{\text {det_n_m }}$ effectively equal to $\operatorname{OTF}(k)$. After achieving the values of $M 1$ and $M 2$, a FFT-based cross-correlation is executed between these two items by using a predicted pattern frequency $k_{0}$, yielding varied correlation values at different pixel-related discrete frequencies. The more accurate peak position in a subpixel scale can be obtained by a parabolic interpolation fitting of these cross-correlation values. To achieve the optimal $k_{0}$, an iterative optimization process aiming to maximize the peak correlation value can be carried out, to refine the peak position by applying a subpixel frequency space shift to the current $k_{0}$ in the form of real-space phase gradients. The accurate position of this maximized cross-correlation peak, as discussed above, yields the precise pattern frequency $k_{0}$ as well as its direction.

By setting $m=1$ or -1 in $M 1=c_{m} e^{i m \varphi_{n}} \cdot M 2$, both the initial phase $\varphi_{n}$ and the pattern contrast $c_{m}$ can be retrieved by a complex linear regression of $M 1$ against $M 2$ at the optimal pattern frequency $k_{0}$. This phase retrieval process is similar with the cross-correlation method in Sec. 6, however in which, the initial phase is further optimized by changing the phase values in an iteration process.

Although the parameters $c_{m}, k_{0}$ and $\varphi_{n}$ required to resolve the spectral terms $\tilde{E}_{m}(k)$ have been obtained up to date, one more step of image deconvolution must be executed to reconstruct the final super-resolution image $\tilde{S}(k)$, as shown in Eqs. (7) and (8). Executing an inverse filter of OTF on both sides of Eq. (8) looks like a straightforward method to obtain $\tilde{S}(k)$, but it will fail for noisy image data because of the over-amplification of the noise. In practice, a generalized Wiener filter is used in the image deconvolution process ${ }^{8}$ :

$$
S(k)=\frac{\sum_{m} \mathrm{OTF}^{*}\left(k+m k_{0}\right) \tilde{E}_{m}\left(k+m k_{0}\right)}{\sum_{m}\left|\mathrm{OTF}\left(k+m k_{0}\right)\right|^{2}+w^{2}} A(k),
$$

where $w^{2}$ is the constant Wiener parameter and determined empirically; $A(k)$ is an apodization

Image reconstruction flowchart of SIM

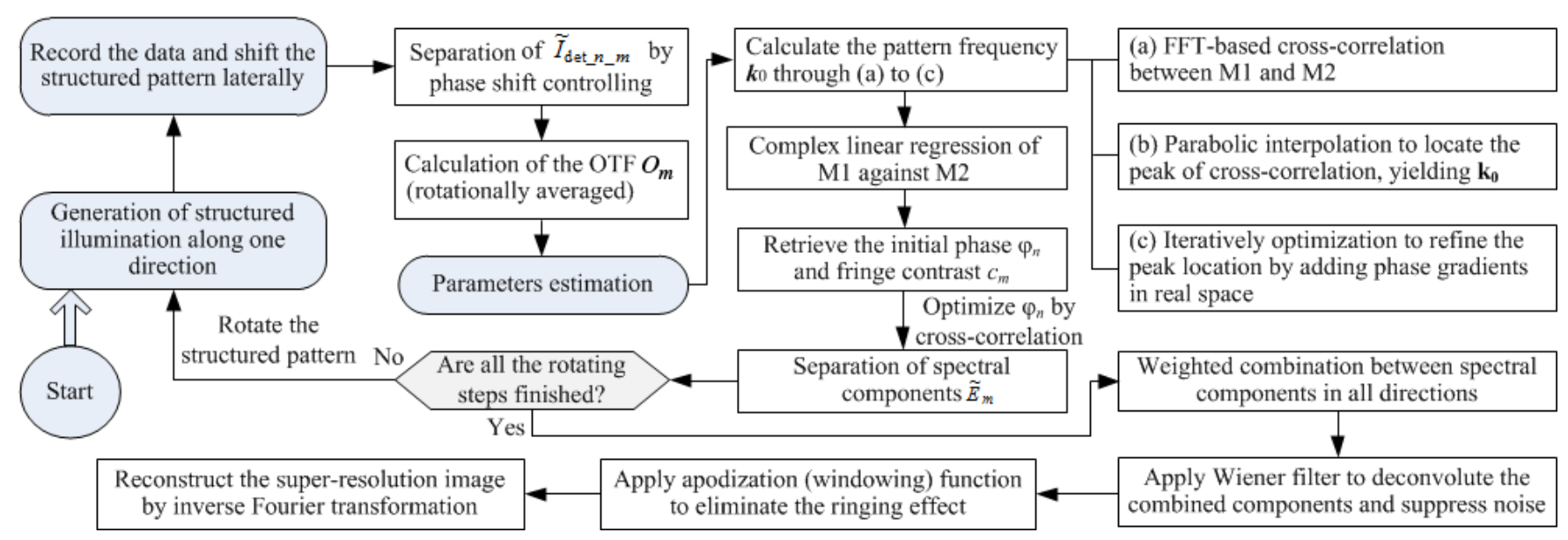

Fig. 8. Flowchart of image reconstruction process in SIM. 
function such as a triangle function or an Euclidean function, to eliminate the ring effect in reconstruction. For the sake of simplicity, each $c_{m} e^{i m \varphi_{n}}$ in Eq. (7) has been absorbed into the $m$ th $\operatorname{OTF}(k)$. By using the Wiener filter function $\mathrm{OTF}^{*}\left(k+m k_{0}\right)$ $A(k) /\left(\sum_{m}\left|\mathrm{OTF}\left(k+m k_{0}\right)\right|^{2}+w^{2}\right)$, the sample information $\tilde{S}(k)$ will be reconstructed. Compared with the inverse filter of OTF, Wiener filter is based on minimizing the mean square error between the reconstructed $\tilde{S}(k)$ and its ideal value, so that is free from white noises amplification. It is worth noting that $O T F(k)$ in Eq. (16) should be weighted averaged in Fourier domain before applying the Wiener filter, given as $\mathrm{OTF}(k)=\sum_{m} \mathrm{OTF}^{2}\left(k+m k_{0}\right) /$ $\sum_{m} \mathrm{OTF}\left(k+m k_{0}\right)$, to further increase the SNR by maximizing the ratio of total transfer strength to total noise strength in the OTF region. The final reconstructed image can be obtained by an inverse Fourier transformation of $\tilde{S}(k)$. The flowchart of the whole image reconstruction process in SIM is drawn in Fig. 8, in which the cross-correlation method is used for phase retrieval.

Optical aberrations in SIM, such as refractive index mismatch and inhomogeneous distributions of small sample features, will reduce the strength of frequency response in the support region of OTF especially at high frequencies, resulting in artifacts in the reconstructed image even at high SNR. To solve this problem, by combining adaptive optics with SIM to compensate the aberrations, the image resolution beyond the diffraction limit can be obtained through thick tissue. ${ }^{28-30}$

\section{Artifacts Sources in Image Reconstruction}

The main artifacts are normally produced by incorrect separation (or resolving) of spectral components $\tilde{S}_{m}\left(k-m k_{0}\right)$ in Eq. (5), which could be caused by imprecise knowledge of pattern phase or pattern contrast, especially under large pattern phase deviations or nonequidistant pattern stepping. ${ }^{16}$ After successful separation of spectral components is obtained, the pattern frequency should be calculated accurately, otherwise these components will be shifted to incorrect positions in Fourier domain, resulting in serious image artifacts especially in the regime of high frequencies.

Another kind of artifact comes from the direct combination of spectral components in post-process under uncorrelated white noise background. When a component of very weak OTF transfer strength (at the edge of its OTF) is superimposed to a component of high OTF transfer strength (at the center of its OTF), only little sample information is added, meanwhile the additional noise (independent with OTF) of this component will increase the overall noises. To avoid this, the weighted averaging between different components should be carried out before combination to make each component contribute to the final reconstructed image in such a way — the ratio of total transfer strength to total noise is maximal.

The direct deconvolution using an inverse filter in noise background will also produce artifact. Although this deconvolution would indeed yield the best results in a noise-free condition, it absolutely fails for noisy images. If the transfer strength of weighted averaging OTF is low, dividing by it as carried out in the inverse filter excessively magnifies noises present at these frequencies. In contrast, the Wiener filter can solve this problem by minimizing the mean square error between the reconstructed sample image and the true image. In addition, the hard edges of calculated OTF which might result from Wiener-filtering and produce artifacts, can be avoided by applying an apodization function as shown in Eq. (16).

Finally, the strongly scattering of thick samples will lead to a distortion of the pattern fringes, which can be partially overcome by combining SIM with adaptive optics. ${ }^{30}$ And the SIM system should be set up stably to avoid the sample movement or beamjitter in optical path.

\section{Conclusion}

Under structured illumination, some initially undetectable sample spectra are optically encoded into the detectable regime in SIM. After being computationally decoded, the overlapped sample spectra are shifted to their correct spectral positions to extend the effective support region of OTF in Fourier domain, finally resulting in a reconstructed image with about two-fold enhanced spatial resolution. In practice, the generation of structured illumination and the data post-processing are two essential issues in SIM. In image recording process, we simulate the PSF and OTF of a conventional microscope, which exclusively contributes to the image 
blur. As coherent illumination is crucial for good pattern contrast of very fine fringes, a structured pattern is generated via the interference of two simulated coherent Gaussian beams. In the complex post-process of reconstruction, firstly three different methods to retrieve the initial pattern phases are sequentially introduced and compared. Secondly, a cross-correlation optimization method is used to retrieve the optimal pattern frequency and the initial phase in the noisy raw image with fine illumination pattern, while the pattern contrast is obtained by measuring the light intensity variance on a fluorescent bead. Thirdly, the spectral components are separated and shifted to their original positions in Fourier domain, resulting in extended detectable spectra. Finally, an image deconvolution process is executed by using a Weiner filter function in which the OTF of microscope is weighted averaged, ultimately achieving the final noise-free superresolution image. The analysis and testing verify the auto-correlation algorithm affords the fast, most precise and robust measurement of initial pattern phases. In addition, the blind-SIM using unknown illumination patterns and the basic fundamental of nonlinear SIM are briefly introduced. The detailed simulation model, the programming flowchart and the analysis of artifacts sources can be a helpful referable guide to the image reconstruction of SIM in experiments.

\section{Acknowledgment}

This work is supported by National Natural Science Foundation of China (Nos. 61361160418 and $61327902)$.

\section{References}

1. L. Schermelleh, R. Heintzmann, H. Leonhardt, "A guide to super-resolution fluorescence microscopy," J. Cell Biol. 190(2), 165-175 (2010).

2. B. Huang, H. Babcock, X. Zhuang, "Breaking the diffraction barrier: Super-resolution imaging of cells," Cell 143(7), 1047-1058 (2010).

3. M. Maglione, S. J. Sigrist, "Seeing the forest tree by tree: Super-resolution light microscopy meets the neurosciences," Nat. Neurosci. 16, 790-797 (2013).

4. L. Shao, P. Kner, E. H. Rego, M. G. L. Gustafsson, "Super-resolution 3D microscopy of live whole cells using structured illumination," Nat. Methods 8, 1044-1046 (2011).
5. D. Dan, M. Lei, B. L. Yao, W. Wang, M. Winterhalder, A. Zumbusch, Y. J. Qi, L. Xia, S. H. Yan, Y. L. Yang, P. Gao, T. Ye, W. Zhao, "DMD-based LED-illumination super-resolution and optical sectioning microscopy," Sci. Rep. 3, 1116 (2013).

6. R. Heintzmann, C. G. Cremer, "Laterally modulated excitation microscopy: Improvement of resolution by using a diffraction grating," Proc. SPIE 3568, 185-196 (1999).

7. M. G. Gustafsson, "Surpassing the lateral resolution limit by a factor of two using structured illumination microscopy," J. Microsc. 198(2), 82-87 (2000).

8. M. G. Gustafsson, L. Shao, P. M. Carlton, C. J. Wang, I. N. Golubovskaya, W. Z. Cande, D. A. Agard, J. W. Sedat, "Three-dimensional resolution doubling in wide-field fluorescence microscopy by structured illumination," Biophys. J. 94(12), 49574970 (2008).

9. R. Förster, H. W. L. Walther, A. Jost, M. Kielhorn, K. Wicker, R. Heintzmann, "Simple structured illumination microscope setup with high acquisition speed by using a spatial light modulator," Opt. Express 22(17), 20663-20677 (2014).

10. A. G. York, S. H. Parekh, D. D. Nogare, R. S. Fischer, K. Temprine, M. Mione, A. B. Chitnis, C. A. Combs, H. Shroff, "Resolution doubling in live, multicellular organisms via multifocal structured illumination microscopy," Nat. Methods 9, 749-754 (2012).

11. P. Pankajakshan, Z. Kam, A. Dieterlen, G. Engler, L. B. Féraud, J. Zerubia, J. C. O. Marin, Pointspread function model for fluorescence macroscopy imaging, Proc. Asilomar Conf. Signals, Systems and Computers, pp. 1364-1368 (2010).

12. S. F. Gibson, F. Lanni, "Diffraction by a circular aperture as a model for three-dimensional optical microscopy," J. Opt. Soc. Am. A 6(9), 1357-1367 (1989).

13. Y. Hiraoka, J. W. Sedat, D. A. Agard, "Determination of three-dimensional imaging properties of a light microscope system," Biophys J. 57(2), 325-333 (1990).

14. L. M. Hirvonen, K. Wicker, O. Mandula, R. Heintzmann, "Structured illumination microscopy of a living cell," Eur Biophys J. 38(6), 807-812 (2009).

15. D. Dan, B. L. Yao, M. Lei, "Structured illumination microscopy for super-resolution and optical sectioning," Chin. Sci. Bull. 59(12), 1291-1307 (2014).

16. K. Wicker, Super-Resolution Fluorescence Microscopy Using Structured Illumination, Super-Resolution Microscopy Techniques in the Neurosciences, Chap. 7, E. Fornasiero, S. Rizzoli, Eds., pp. 133-165, Humana Press, Göttingen (2014). 
17. P. Kner, B. B. Chhun, E. R. Griffis, L. Winoto, M. G. L. Gustafsson, "Super-resolution video microscopy of live cells by structured illumination," Nat. Methods 6, 339-342 (2009).

18. R. Fiolka, L. Shao, E. H. Rego, M. W. Davidson, M. G. L. Gustafsson, "Time-lapse two-color 3D imaging of live cells with doubled resolution using structured illumination," Proc. Natl. Acad. Sci. 109(14), 5311-5315 (2012).

19. J. Fitzgibbon, K. Bell, E. King, K. Oparka, "Superresolution imaging of plasmodesmata using threedimensional structured illumination microscopy," Plant Physiol. 153(4), 1453-1463 (2010).

20. R. Heintzmann, M. G. L. Gustafsson, "Subdiffraction resolution in continuous samples," Nat. Photon. 3, 362-364 (2009).

21. F. Wei, Z. Liu, "Plasmonic structured illumination microscopy," Nano Lett. 10(7), 2531-2536 (2010).

22. S. A. Shroff, J. R. Fienup, D. R. Williams, "Phase-shift estimation in sinusoidally illuminated images for lateral superresolution," JOSA A 26(2), 413-424 (2009).

23. K. Wicker, O. Mandula, G. Best, R. Fiolka, R. Heintzmann, "Phase optimisation for structured illumination microscopy," Opt. Express 21(2), 20322049 (2013).

24. K. Wicker, "Non-iterative determination of pattern phase in structured illumination microscopy using auto-correlations in Fourier space," Opt. Express 21(21), 24692-24701 (2013).
25. E. Mudry, K. Belkebir, J. Girard, J. Savatier, E. Le Moal, C. Nicoletti, A. Sentenac, "Structured illumination microscopy using unknown speckle patterns," Nat. Photon. 6(5), 312-315 (2012).

26. R. Ayuk, H. Giovannini, A. Jost, E. Mudry, J. Girard, T. Mangeat, N. Sandeau, R. Heintzmann, K. Wicker, K. Belkebir, A. Sentenac, "Structured illumination fluorescence microscopy with distorted excitations using a filtered blind-SIM algorithm," Opt. Lett. 38(22), 4723-4726 (2013).

27. A. Jost, E. Tolstik, P. Feldmann, K. Wicker, A. Sentenac, R. Heintzmann, "Optical sectioning and high resolution in single-slice structured illumination microscopy by thick slice blind-SIM reconstruction," PLoS One 10(7), 0132174 (2015).

28. R. D. Simmonds, M. J. Booth, "Modelling of multiconjugate adaptive optics for spatially variant aberrations in microscopy," J. Opt. 15(9), 094010 (2013).

29. B. Thomas, A. Wolstenholme, S. N. Chaudhari, E. T. Kipreos, P. Kner, "Enhanced resolution through thick tissue with structured illumination and adaptive optics," J. Biomed. Opt. 20(2), 026006 (2015).

30. N. G. Chen, S. Rehman, C. J. R. Sheppard, "Advanced optical microscopy methods for in vivo imaging of sub-cellular structures in thick biological tissues," J. Innov. Opt. Health Sci. 7(5), 1440001 (2014). 